\title{
Erratum
}

\section{Viability and function of primary human endothelial cells on smooth poly (ether imide) films}

C. Schulz, M. von Rüsten-Lange, A. Krüger, A. Lendlein and F. Jung Center for Biomaterial Development and Berlin Brandenburg Center for Regenerative, Therapies, Institute of Polymer Research, Helmholtz-Zentrum Geesthacht, Teltow, Germany

[Clinical Hemorheology and Microcirculation 52(2-4) (2012), 267-282] DOI: DOI 10.3233/CH-2012-1604

The Acknowledgments Section is missing from the article and should be added:

\section{Acknowledgments}

The authors thank the Bundesministerium für Bildung und Forschung (BMBF) for funding within project number 0315696A "Poly4Bio BB"). 